\title{
Circular dichroism in planar extrinsic chirality metamaterial at oblique incident beam
}

\author{
Seoungjun Lee ${ }^{\mathrm{a}, *}$, Zengbo Wang ${ }^{\mathrm{a}, \mathrm{b}}$, Cheng Feng ${ }^{\mathrm{c}}$, Jiao Jiao ${ }^{\mathrm{a}}$, Ashfaq Khan ${ }^{\mathrm{d}}$, Lin Li $^{\mathrm{a}}$ \\ a School of Mechanical, Aerospace and Civil Engineering, University of Manchester, Manchester M13 9PL, UK \\ ${ }^{\mathrm{b}}$ Schools of Electronic Engineering, Bangor University, Dean Street, Bangor, Gwynedd LL57 1UT, UK \\ ${ }^{\mathrm{C}}$ Chemistry Department, University of Leicester, University Road, Leicester LE1 7RH, UK \\ ${ }^{\mathrm{d}}$ Department of Mechanical Engineering, NWFP University of Engineering and Technology, Peshawar 25000, Pakistan
}

\section{A R T I C L E I N F O}

\section{Article history:}

Received 3 May 2013

Received in revised form

10 July 2013

Accepted 11 July 2013

Available online 24 July 2013

Keywords:

Metamaterial

Chirality

Circular dichroism

Visible wavelength

\begin{abstract}
A B S T R A C T
We present a two dimensional planar extrinsic chirality metamaterial (2D-ECM) design that has strong circular dichroism (CD) in the visible spectrum range rather than usual near-infrared and terahertz range. The 2D-ECM is theoretically investigated by incident beam angles and meta-molecules unit sizes in visible spectrums. Physical mechanism was illustrated in figures of vector directions in electric field.
\end{abstract}

(c) 2013 Elsevier B.V. All rights reserved.

\section{Introduction}

Circular dichroism (CD) is characterized by the differential absorption of right circularly polarized (RCP) light and left circularly polarized (LCP) light, and is related to polarization effect which can determine optical activity [1,2]. Circular dichroism spectroscopy has been widely used to gain information about biomolecule, DNA [3-5] and organic compounds [6]. Nanoparticles obtained strong $\mathrm{CD}$ in the geometry and composition of a chiral molecule [7-9]. CD was observed in double layers [10], mutually twisted unconnected layers [11], and single-layered gold sawtooth gratings [12] of chiral planar metamaterials at nearinfrared wavelengths. Using terahertz frequency, optical activity and coupling effects can generate and assist CD [13,14]. A threedimensional chiral metamaterial of mutually twisted planar metal patterns generated giant $C D$ in parallel planes due to negative index of refraction [15]. In non-chiral metamaterials, optimized unit cell structure can improve the peak point of $\mathrm{CD}$ [16], and elliptical nano-holes array allows to observe $C D$ in visible spectrum [17]. In this paper, we report that two dimensional planar extrinsic chirality metamaterial (2D-ECM) can generate strong CD at visible spectrums using electric distribution of Floquet modes in CST (computer simulation technology) microwave studio software. The structure of the 2D-ECM contains a silver single layer structure

\footnotetext{
* Corresponding author. Tel.: +44161306 3816 .

E-mail address: seoungjun.lee@postgrad.manchester.ac.uk (S. Lee).
}

on a borosilicate glass substrate. The refractive index and extinction coefficient of silver is changed by wavelengths, and measured at visible wavelengths [18]. The dielectric material of a borosilicate glass (N-BK7) is used for a thick substrate, and the data of the refractive index is obtained [19].

\section{Methods}

The transmission of RCP and LCP can be mathematically defined in a $2 \times 2 t$-matrix as [20]

$\left(\begin{array}{l}T_{+} \\ T_{-}\end{array}\right)=\left(\begin{array}{ll}t_{++} & t_{-+} \\ t_{+-} & t_{--}\end{array}\right)\left(\begin{array}{l}I_{+} \\ I_{-}\end{array}\right)$

where ' + ' and '-' are symbols for RCP and LCP incident beams, respectively. The matrix is combined by transmission $(T)$ and incident $(I)$ beam. $t_{++}$and $t_{--}$correspond to the transmission amplitude of RCP $\left(I_{+}\right)$and LCP $\left(I_{-}\right)$incident beams. $t_{+-}$and $t_{-+}$are calculated by the conversion between RCP and LCP. The incident electric field $\left(E^{\text {in }}\right)$ and transmission electric field $\left(E^{\text {out }}\right)$ are related to the $t$-matrix, where LCP and RCP indicate subscripts+and - as defined [20]:

$E_{i}^{o u t}=t_{i j} E_{j}^{i n}$

and the Circular dichroism (CD) of LCP and RCP is mathematically defined as $[20,21]$

$\mathrm{CD}=\left|t_{++}\right|^{2}-\left|t_{--}\right|^{2}$ 


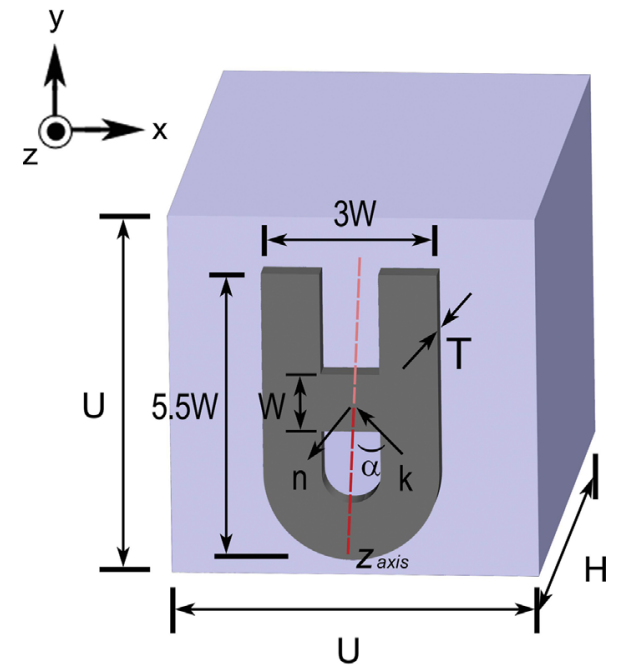

Fig. 1. Asymmetric unit cell structure $(U=250 \mathrm{~nm})$ of a borosilicate glass substrate $(H=1000 \mathrm{~nm})$ and silver unit cells $(T=20 \mathrm{~nm}$ and $W=40 \mathrm{~nm}) . n$ is the direction of vector perpendicular to the unit cell. $k$ is the direction of the incident beam, and emits along the relative $x$ axis. $\alpha$ is angle of the incident beam, and is calculated by the direction of incident beam $k$ to $z$ axis.

$$
\mathrm{CD}=\left|T_{L C P}\right|-\left|T_{R C P}\right|
$$

CD spectrum was simulated by modern finite integrate technique (FIT) in frequency domain. Different angles of oblique incident beams were applied in the unit cell. The spectrum was demonstrated and compared between LCP and RCP. The 2D-ECM design consists of unit cells of $20 \mathrm{~nm}$ thick silver layer on a $1000 \mathrm{~nm}$ thick borosilicate glass substrate as shown in Fig. 1. The borosilicate glass substrate is dielectric substrate which can help to break mirror symmetry so optical activity can be boosted [22]. At vertical incident beam $\left(\alpha=0^{\circ}\right)$, it forms mirror image because the asymmetric unit cell structure has the same direction as incident beam so it is not 3D-chiral. However, at tilted incident beam $\left(\alpha \neq 0^{\circ}\right)$, the asymmetric unit cell structure is transformed into extrinsically 3D-chiral as it is not mirror image between LCP and RCP [13]. The 2D-ECM forms unit cell boundary condition in $x$ and $y$ dimensions, and open boundary condition in $z$ dimension. The unit cell boundary condition is quite similar to the periodic boundary condition but when open boundaries are perpendicular such as using waveguide ports, the unit cell boundary condition approaches Floquet modes. Oblique incident beams of LCP and RCP propagates into $z$ dimension using waveguide ports. The waveguide ports support to calculate returning power using S-parameters.

\section{Results and discussion}

Fig. 2 shows CD spectrum of the $250 \mathrm{~nm}$ unit cell in wavelengths between $450 \mathrm{~nm}$ and $800 \mathrm{~nm}$ at various incident angles of $\alpha=0^{\circ}, \pm 20^{\circ}, \pm 71^{\circ}$ and $\pm 80^{\circ}$. In wavelengths below $610 \mathrm{~nm}$, the spectrum curves are highly oscillating. This is because of strong optical diffraction associated with the unit cell [20], which may lead to disturbance and diffraction of the oblique incident beams in the layer of the unit cell. Above $610 \mathrm{~nm}$ wavelengths, the spectrum curves change smoothly because the optical diffraction is negligible. The maximum CD peaks were 0.4914 at $726.39 \mathrm{~nm}$ visible wavelength occurring at angles $\alpha=71^{\circ}$, where is the highest difference between LCP and RCP. It shows that $71^{\circ}$ tiled incident beams of LCP and RCP may have distinctive interactions between electric and magnetic dipoles, which may generate most clear optical activity. $C D$ did not appear at $0^{\circ}$ oblique incident beams because the projection of electric and magnetic moment is symmetrically the same between LCP and RCP [23]. It means that the location and magnitude of electric and magnetic dipoles could be the same. The $\pm 20^{\circ}, \pm 71^{\circ}$ and $\pm 80^{\circ}$ were intersected

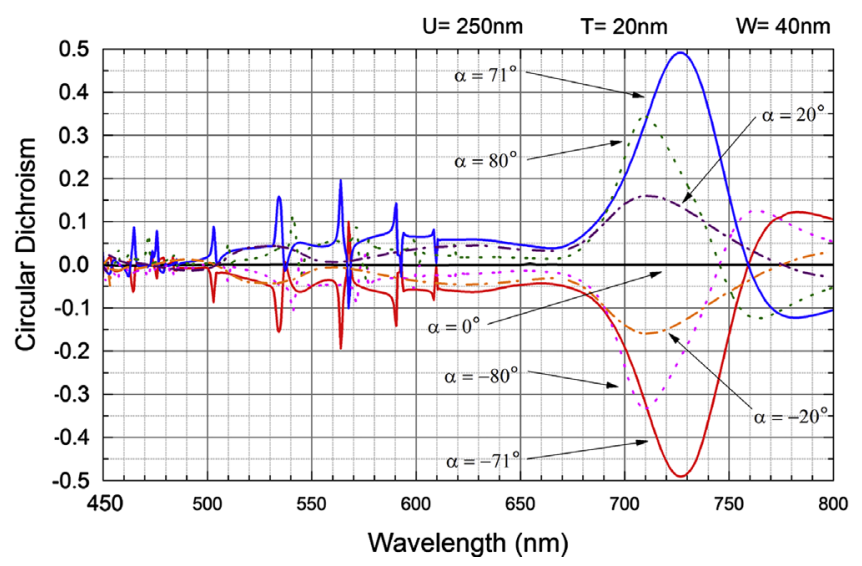

Fig. 2. Comparison of $\mathrm{CD}$ in the $250 \mathrm{~nm}$ unit cell between different incident beam angles as $\alpha= \pm 80^{\circ}, \pm 71^{\circ}, \pm 20^{\circ}$ and $0^{\circ}$ at wavelengths $(\lambda)$ between $450 \mathrm{~nm}$ and $800 \mathrm{~nm}$. The maximum CDs are in Table 1 .

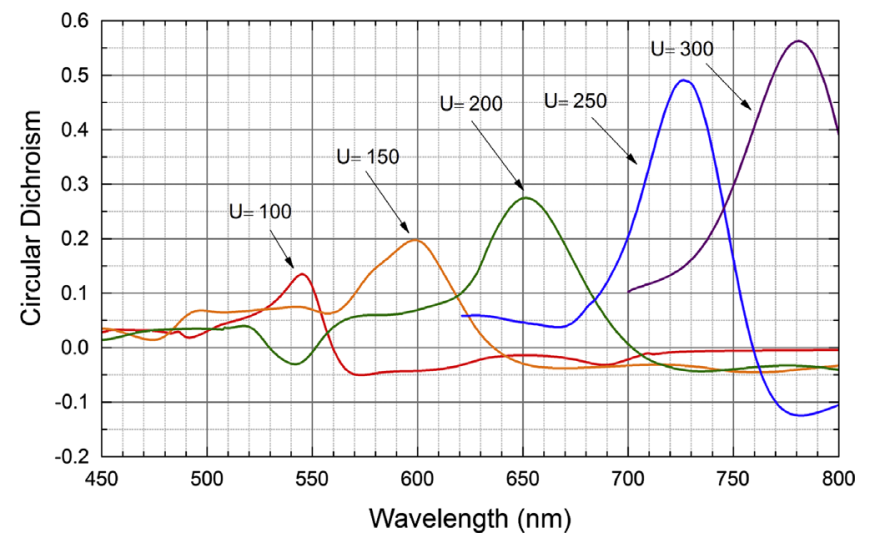

Fig. 3. Dependence of $C D$ on the wavelengths $(\lambda)$ between $450 \mathrm{~nm}$ and $800 \mathrm{~nm}$ for $100 \mathrm{~nm}$ unit cell with $\alpha=77^{\circ}, 150 \mathrm{~nm}$ unit cell with $\alpha=58^{\circ}, 200 \mathrm{~nm}$ unit cell with $\alpha=45^{\circ}, 250 \mathrm{~nm}$ unit cell with $\alpha=71^{\circ}$, and $300 \mathrm{~nm}$ unit cell with $\alpha=57^{\circ}$. The maximum CDs are in Table 2. Highly oscillated curves are removed in $U=250$ and 300 .

Table 1

aaaaa250 nm unit cell with various angles, wavelengths and CD.

\begin{tabular}{rll}
\hline Angle $(\alpha$, deg) & Optimized wavelength $(\lambda, \mathrm{nm})$ & Transmission spectrum $(\mathrm{CD})$ \\
\hline 0 & It does not appear & It does not appear \\
20 & 710.90 & 0.1602 \\
71 & 726.36 & 0.4914 \\
80 & 710.06 & 0.3438 \\
\hline
\end{tabular}

Table 2

Different sizes of unit cells with optimized angles, wavelengths and CD.

\begin{tabular}{llll}
\hline $\begin{array}{l}\text { Unit cell size } \\
(U, \mathrm{~nm})\end{array}$ & $\begin{array}{l}\text { Optimized angle } \\
(\alpha, \mathrm{deg})\end{array}$ & $\begin{array}{l}\text { Optimized } \\
\text { wavelength }(\lambda, \mathrm{nm})\end{array}$ & $\begin{array}{l}\text { Transmission } \\
\text { spectrum }(\mathrm{CD})\end{array}$ \\
\hline 100 & 77 & 545.45 & 0.1345 \\
150 & 58 & 598.80 & 0.1976 \\
200 & 45 & 651.47 & 0.2750 \\
250 & 71 & 726.36 & 0.4914 \\
300 & 57 & 781.25 & 0.5627 \\
\hline
\end{tabular}


and no $C D$ value at $770 \mathrm{~nm}, 760 \mathrm{~nm}$, and $740 \mathrm{~nm}$ wavelengths, respectively. At intersected wavelength points, the magnitude of LCP and RCP could be quite similar so CD was not generated. In addition, three different angles of $\mathrm{CD}$ can have similar curve

Table 3

Different widths of $250 \mathrm{~nm}$ unit cell with optimized angles, wavelengths and CD.

\begin{tabular}{llll}
\hline $\begin{array}{l}\text { Width } \\
(W)\end{array}$ & $\begin{array}{l}\text { Optimized angle } \\
(\alpha, \mathrm{deg})\end{array}$ & $\begin{array}{l}\text { Optimized wavelength } \\
(\lambda, \mathrm{nm})\end{array}$ & $\begin{array}{l}\text { transmission } \\
\text { spectrum }(\mathrm{CD})\end{array}$ \\
\hline 24 & 63 & 599.63 & 0.1878 \\
28 & 55 & 621.30 & 0.2635 \\
32 & 45 & 650.05 & 0.2762 \\
40 & 71 & 726.36 & 0.4914 \\
\hline
\end{tabular}

patterns but the peak and intersected position were various. It might cause optical activity with particular oblique incident beams. Different wavelengths of oblique incident beams can determine different locations of electric and magnetic diploes while $k$ vector penetrates into the extrinsically 3D-chiral.

A comparison between $100 \mathrm{~nm}, 150 \mathrm{~nm}, 200 \mathrm{~nm}, 250 \mathrm{~nm}$, and $300 \mathrm{~nm}$ unit cells is presented in Fig. 3. It demonstrates that the different sizes of unit cells can determine the peak position of a wavelength and magnitude of $\mathrm{CD}$. When the unit cell size is shrunk, the maximum CD is decreased and the peak wavelength is shifted to smaller wavelengths. Otherwise, increasing unit cell size generates the reverse effect. The effects may cause different electric and magnetic moments on the surface of the 2D-ECM. The bigger unit cell $(U)$ and wider structure $(W)$ can generate

\section{Position (nm)}
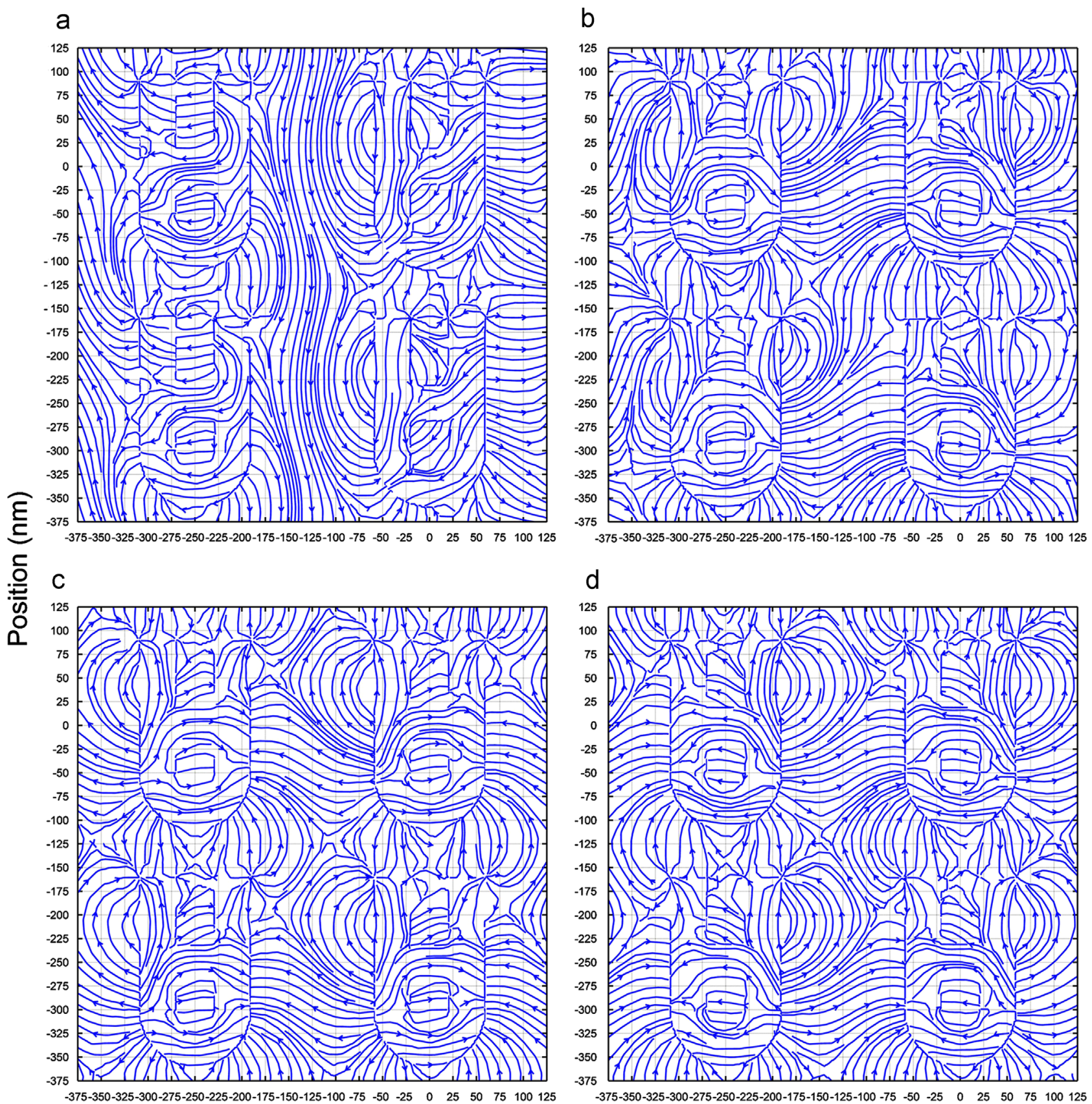

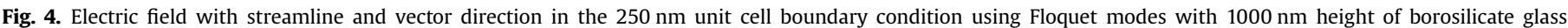
substrate, $T=20 \mathrm{~nm}$ thickness, and $W=32 \mathrm{~nm}$ width of silver at (a), (b) $\alpha=71^{\circ}$ and (c), (d) $0^{\circ}$ of incident beam, with $\lambda=726.36 \mathrm{~nm}$ between LCP and RCP. 
higher electric and magnetic energies because the optical activity can have more active resulting in the electric and magnetic diploes. Furthermore, the position and power of magnetic and electric dipoles may be relocated at diverse angles of oblique incident beams. As a result, the magnitude of LCP and RCP is not the same in the 2D-ECM. The $300 \mathrm{~nm}$ unit cell has the biggest CD but it is out of the visible spectrum so the $250 \mathrm{~nm}$ unit cell has the biggest $C D$ in visible spectrum. Various unit cell sizes with Maximum CDs and these of diverse angles are presented in Table 2. It is an agreeable result that the 2D-ECM can generate giant $\mathrm{CD}$ compared to planar chiral split-ring metamaterials $(\mathrm{CD}=0.04$ in $\mathrm{GHz})$ [21] and planar non-chiral elliptical nanoholes array metamaterials $(C D=0.12$ in visible spectrum) [17]. The $100 \mathrm{~nm}, 150 \mathrm{~nm}, 200 \mathrm{~nm}, 250 \mathrm{~nm}$, and $300 \mathrm{~nm}$ unit cells have the optimized angles at $\alpha=77^{\circ}, 58^{\circ}, 45^{\circ}, 71^{\circ}$, and $57^{\circ}$, respectively. Optimized angles are dependent of unit cell sizes because the electric and magnetic dipoles may be located in different positions, so different forces of optical activity may be generated in the varied unit cells. The $250 \mathrm{~nm}$ unit cell is demonstrated with varied width of the ECM in Table 3. It includes maximum CDs, that of an angle and a wavelength. It shows that optimized unit cell structure can control a peak and that of a wavelength of $\mathrm{CD}$. The maximum CDs are $0.1878,0.2635,0.2762$, and 0.4914 at $W=24,28,32$, and 40 , respectively. The narrow width can shrink the total size of silver unit cells so that the area of dipoles may be diminished. As a result, the wavelength of maximum $\mathrm{CDs}$ can be determined by unit cell size, and the bigger unit cell size can shift to higher wavelength. The area of the 2D-ECM can affect the wavelength of $C D$ and $C D$ value. The larger area could have strong $C D$ but the wavelength of $C D$ could be also increased. Strong optical activity may not generate in the narrow width and small size of unit cells as it may reduce the energy of surface plasmon on the 2D-ECM. Thus, $U=250$ and $W=40$ can be optimized CDs in visible spectrum.

A comparison of LCP and RCP is presented in electric field using CST raw data with Matlab coding as shown in Fig. $4.71^{\circ}$ tilted and vertical incident beam was applied in electric field. The four unit cells were monitored simultaneously between LCP and RCP. In the vertical incident beam $\left(0^{\circ}\right.$ oblique incident beam $)$, the LCP states of unit cells are symmetrically opposite to that of RCP. It means that the electric interaction of unit cells is almost same between LCP and RCP so optical activity did not appear in CD spectrum. In $71^{\circ}$ tilted incident beam, the distribution of positive and negative charges is different in LCP and RCP. Such as the row of unit cells is quite same but the column of unit cells has different locations in positive and negative charges. As a result, CD is obtained by trapped energy in resonance during polarization conversation [24]. The electric interaction between LCP and RCP can generate different electric flows in the unit cells. Thus, giant $C D$ can be generated by the different energy distribution between LCP and RCP in tilted incident beams. It can be also related to different magnitudes of $C D$, and that of an optimized angle and a wavelength. Furthermore, The unit cell structure can affect actual coupling aspects [25] so the interaction between neighboring unit cells may cause main reason of strong $C D$.

\section{Conclusions}

In visible spectrum, we show that the 2D-ECM can have strong $\mathrm{CD}$ at angled incident beam due to critical interactions between positive and negative charges in LCP and RCP. The strong CD may be obtained by different neighboring interactions of unit cells between LCP and RCP. In vertical incident beam, CD is not generated because the interactions of unit cells are identically same as LCP and RCP. The size of unit cell $(U)$ and width $(W)$ can increase the wavelength and magnitude of $C D$ but it will lead out range of visible spectrum. The $\mathrm{CD}$ of the $2 \mathrm{D}-\mathrm{ECM}$ is significantly affected by incident beam angles and meta-molecules unit sizes. Moreover, such strong CD can open up bio-molecular detecting systems and vibration sensors at visible spectrums because the detecting system works better with strong CD. Thus, sensitive biological samples may be applied in the 2D-ECM such as the identification of DNA or virus.

\section{References}

[1] C. Menzel, C. Rockstuhl, F. Lederer, Physical Review A: Atomic, Molecular, and Optical Physics 82 (2010).

[2] S.N. Volkov, K. Dolgaleva, R.W. Boyd, K. Jefimovs, J. Turunen, Y. Svirko, B. K. Canfield, M. Kauranen, Physical Review A-Atomic, Molecular, and Optical Physics 79 (2009).

[3] S. Paramasivan, I. Rujan, P.H. Bolton, Methods 43 (2007) 324.

[4] L. Whitmore, B.A. Wallace, Biopolymers 89 (2008) 392.

[5] N.J. Greenfield, Nature Protocols 1 (2006) 2876.

[6] N. Berova, L.D. Bari, G. Pescitelli, Chemical Society Reviews 36 (2007) 914.

[7] Z. Fan, A.O. Govorov, Nano Letters 10 (2010) 2580.

[8] J. George, K. George Thomas, Journal of the American Chemical Society 132 (2010) 2502

[9] A.O. Govorov, Z. Fan, P. Hernandez, J.M. Slocik, R.R. Naik, Nano Letters 10 (2010) 1374.

[10] D.H. Kwon, P.L. Werner, D.H. Werner, Optics Express 16 (2008) 12802.

[11] E. Plum, V.A. Fedotov, A.S. Schwanecke, N.I. Zheludev, Y. Chen, Applied Physics Letters 90 (2007).

[12] W. Gao, C.Y. Ng, H.M. Leung, Y. Li, H. Chen, W.Y. Tam, Journal of the Optical Society of America B: Optical Physics 29 (2012) 3021.

[13] R. Singh, E. Plum, W. Zhang, N.I. Zheludev, Optics Express 18 (2010) 13425

[14] M. Decker, R. Zhao, C.M. Soukoulis, S. Linden, M. Wegener, Optics Letters 35 (2010) 1593.

[15] E. Plum, J. Zhou, J. Dong, V.A. Fedotov, T. Koschny, C.M. Soukoulis, N I. Zheludev, Physical Review B-Condensed Matter and Materials Physics 79 (2009).

[16] C. Feng, Z.B. Wang, S. Lee, J. Jiao, L. Li, Optics Communications 285 (2012) 2750

[17] T. Cao, M.J. Cryan, Journal of Electromagnetic Waves and Applications 26 (2012) 1275

[18] E.D. Palik, Handbook of Optical Constants of Solids, Academic Press, Boston, 1985.

[19] Optical Glass Data Sheets, Schott, 2013.

[20] E. Plum, X.X. Liu, V.A. Fedotov, Y. Chen, D.P. Tsai, N.I. Zheludev, Physical Review Letters 102 (2009).

[21] A.V. Novitsky, V.M. Galynsky, S.V. Zhukovsky, Physical Review B-Condensed Matter and Materials Physics 86 (2012).

[22] S.I. Maslovski, D.K. Morits, S.A. Tretyakov, Journal of Optics A: Pure and Applied Optics, 11, , 2009.

[23] E. Plum, V.A. Fedotov, N.I. Zheludev, Journal of Optics A: Pure and Applied Optics 11 (2009).

[24] S.V. Zhukovsky, A.V. Novitsky, V.M. Galynsky, Optics Letters 34 (2009) 1988.

[25] J. Petschulat, A. Chipouline, A. Tünnermann, T. Pertsch, C. Menzel, C. Rockstuhl T. Paul, F. Lederer, Physical Review B-Condensed Matter and Materials Physics 82 (2010). 